\title{
Dogville y la sociedad educadora: apuntes para una lectura desde las otras morales*
}

//Dogville and education society: Reading other moral codes

Investigaciones $\quad \begin{aligned} & \text { de Educación, N. } 63 . \\ & \text { Segundo semestre de 2012, }\end{aligned}$

//Dogville e a sociedade educadora:

apontamentos para uma leitura a partir

das outras morais

David Andrés Rubio Gaviria**

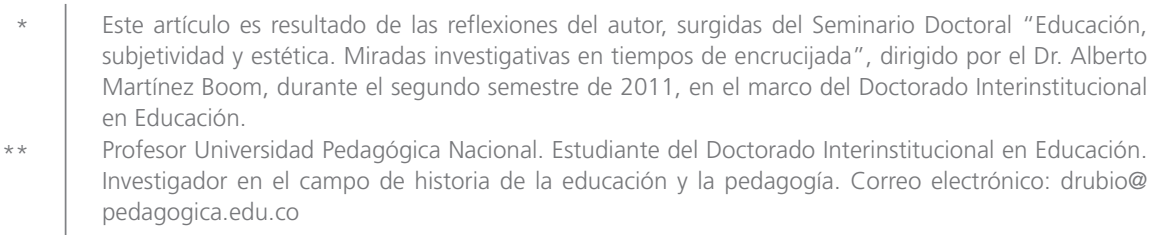

\section{Resumen}

Este artículo propone una reflexión, inicialmente metodológica, alrededor del problema del origen de la moral, para situar allí una perspectiva que favorece la introducción de la tesis consistente en que en el mundo contemporáneo es más preciso discutir a propósito de la emergencia de "las morales", en plural, en vez de acusar un origen, como ampliamente se discute en el proyecto genealógico propuesto por Nietzsche. Se propone, a su vez, la idea de la sociedad educadora, en el ámbito de la planetización del mundo actual, toda vez que las preguntas por la moral se constituyen en factores de contingencia en el debate. Finalmente, se acude a la obra cinematográfica Dogville, del director danés Lars von Trier, como pretexto clave para la convergencia de las ideas que se exponen a lo largo del texto.

\section{Abstract}

This paper initially offers a methodological reflection around the morality issue from its origin in order to give a perspective promoting a thesis where a discussion in the contemporary world about emergence of plural "moral codes" is required instead of a source widely acknowledged and discussed by Nietzsche in his genealogical principle. There is also a proposal of education society in world globalization of today since moral questions typify contingency factors in the relevant debate. Finally, Dogville by Danish director Lars von Trier is a key pretext for blending ideas throughout the text.

\section{Resumo}

O artigo propõe uma reflexão, inicialmente metodológica, em torno do problema da origem da moral, para situar ali uma perspectiva que favorece a introdução da tese consistente de que no mundo contemporâneo é mais preciso discutir a propósito da emergência das "morais", no plural, antes de acusar uma origem, como amplamente se discute no projeto genealógico proposto por F. Nietzsche. Propõe, por sua vez, a ideia de sociedade educadora, no âmbito da planetarização do mundo atual, toda vez que as perguntas sobre a moral se constituem em fatores de contingência no debate. Finalmente, se reporta à obra cinematográfica Dogville, do diretor dinamarquês Lars Von Trier, como pretexto-chave para a convergência das ideias expostas ao longo do texto.

\section{Palabras Clave}

Moral, genealogía, sociedad educadora, planetización, transvaloración.

\section{Keywords}

Moral, genealogy, education society, globalization, transvaluation.

\section{Palavras chave}

Moral, genealogia, sociedade educadora, planetarização, transvalorização 
En La genealogía de la moral, Nietzsche (2009) se resiste al modo de hacer historia de los historiadores. Incluso, ve con desdeño la reflexión de psicólogos que, como Paul Ree, apuestan por una historia de la moral, que incluye la noción del origen. A propósito de esta "resistencia", Michel Foucault recuerda al filósofo alemán, en un sarcasmo no muy fácil de interpretar, a juicio del propio Foucault:

En algún punto perdido del universo, cuyo resplandor se extiende a innumerables sistemas solares, hubo una vez un astro en el que unos animales inteligentes inventaron el conocimiento. Fue aquel el instante más mentiroso y arrogante de la historia universal. (Nietzsche, citado por Foucault, 1996, p. 12).

En efecto, el conocimiento es inventado. $Y$ es inventado porque no acusa un origen. El conocimiento, como la moral, o los sentimientos, es resultado de luchas, de fuerzas, como sugiere el propio Nietzsche. No podemos esperar que la historia nos dé cuenta de esas luchas, al menos esa historia que se ocupa, como diría Foucault (2007), de hallar explicaciones al presente desde la noción de los universales. Para Foucault, la historia que parte de la existencia de universales, con frecuencia hace el camino a la inversa, al intentar mostrar los procesos humanos, en lo que los detalles son el lugar último, una especie de punto de llegada. Al contrario, nos dirá Foucault, la investigación histórica de otro carácter, la contrahistoria, es aquella que justamente se fija en el detalle y al margen de los universales. Las fuerzas a las que hiciera alusión Nietzsche, esos puntos de tensión, son precisamente constitutivos del detalle, de eso que había pasado desapercibido, no de los universales a los que acostumbra la historia canónica.

Allí aparecen, en el orden de las ideas de Nietzsche, los conceptos de emergencia (Entstehung) y procedencia (Herkunft), por oposición a la idea del origen. Nos dice Foucault en su lectura, digamos metodológica, sobre la obra de Nietzsche, que

Herkunft: es la fuente, la procedencia; es la vieja pertenencia a un grupo -el de sangre, el de tradición, el que se establece entre aquellos de la misma altura o de la misma bajeza- [...] el análisis de la procedencia permite disociar al yo y hacer pulular, en los lugares y plazas 
de su síntesis vacía, mil sucesos perdidos hasta ahora [...] La procedencia permite también encontrar bajo el aspecto único de un carácter, o de un concepto, la proliferación de sucesos a través de los cuales (gracias a los que, contra los que) se han formado. La genealogía no pretende remontar el tiempo para restablecer una gran continuidad por encima de la dispersión del olvido. Su objetivo no es mostrar que el pasado está todavía ahí bien vivo en el presente, animándolo aún en secreto después de haber impuesto en todas las etapas del recorrido una forma dibujada desde el comienzo. Nada que se asemeje a la evolución de una especie, al destino de un pueblo. Seguir la filial compleja de la procedencia es al contrario mantener lo que pasó en la dispersión que le es propia: es percibir los accidentes, las desviaciones ínfimas -O al contrario los retornos completos-, los errores, los fallos de apreciación, los malos cálculos que ha producido aquello que existe y es válido para nosotros; es descubrir que en la raíz de lo que conocemos y de lo que somos no están en absoluto la verdad ni el ser, sino la exterioridad del accidente. Por esto sin duda todo origen de la moral, desde el momento en que no es venerable -y la Herkunft no lo es nunca- se convierte en crítica (pp. 12-13).

La procedencia de algo, en este caso de la moral, no obedece a encuentros fortuitos, ni tan siquiera sistemáticos, con la verdad de las cosas. Es en el accidente, dirá Foucault (1979), en aquella exterioridad, aquella superficie, en donde atisbaremos lo que existe, no ya su verdad en un estado esencial -y la esencia, por supuesto, como otra idea que se pone en cuestión-. La procedencia como un resultado de multiplicidad de fuerzas y que no se debe sino al azar. Así, continuará Foucault (1979): "la búsqueda de la procedencia no funda, al contrario: remueve aquello que se percibía inmóvil, fragmenta lo que se pensaba unido; muestra la heterogeneidad de aquello que se imaginaba conforme a sí mismo" (p. 13).

La emergencia, por otro lado, es el instante de la aparición. Las cosas tienen procedencias en plural; la emergencia es la materialidad de ese encuentro de fuerzas que identificamos como procedencias:

La emergencia es pues, la entrada en escena de las fuerzas; es su irrupción, el movimiento de golpe por el que saltan de las 
bambalinas al teatro, cada una con el vigor y la juventud que le es propia. [...] Mientras que la procedencia designa la cualidad de un instinto, su grado o su debilidad, y la marca que este deja en un cuerpo, la emergencia designa un lugar de enfrentamiento [...] Nadie es pues responsable de una emergencia, nadie puede vanagloriarse; esta se produce siempre en el intersticio (p. 17).

La procedencia es una marca, una huella que representa los vestigios de los modos múltiples en que algo se muestra, como la moral, por ejemplo. La emergencia es el instante que nos hace romper con las ideas de autor y de obra como diría Foucault (2004) en su Arqueología del saber, pues "nadie es responsable de una emergencia" y "nadie puede vanagloriarse; esta se produce siempre en el intersticio".

$\mathrm{Y}$ es que precisamente hay otra y otras morales: esa es la noción que deja el trabajo genealógico de Nietzsche, pues tal vez el acento no es necesariamente el haber observado de modo contundente la flacidez de las propuestas de los historiadores de la moral o los psicólogos, sino el modo en que hizo aparecer la pregunta por la moral; la clave del planteamiento reside en el hecho de iniciar preguntándose por el origen de la moral, para terminar en la pregunta por cuáles son las condiciones que hicieron que los hombres formularan los juicios de valor bueno y malvado; se trata de una cuestión que ahonda tanto en la procedencia, como en la emergencia. La pregunta no es entonces por una aparición mágica o divina, si se quiere, sino por las condiciones de los hombres.

\section{Moral, planetización y fin del mundo}

Los asuntos de la moral hoy, son los asuntos del buen ciudadano. En una era de planetización, más que de globalización, como dirían Morin y Baudrillard (2004), las cuestiones de la humanidad se dirimen alrededor de las preguntas sobre cómo es que debe ser habitado el planeta. Planetización como un efecto de las últimas décadas, producido en los procesos de colonización/descolonización -esclavitud/abolición, siempre desde el seno de Occidente-. En la planetización, regulada por los ritmos del mercado, las ideas de "lo bueno", se relacionan con éticas y estéticas del cuidado de sí, de los otros, y del planeta. Todo al servicio de una idea del todos; de un colectivo, que en la lógica de la comunidad, requiere habitar un espacio saludable, un 
medio ambientalmente adecuado $y$, por demás, sostenible y sustentable. Lo bueno, lo moralmente aceptable, es aquello que va en procura de no hacernos daño a nosotros mismos ni a los otros. Se trata de la lógica del cuidado. Es la moral del cuidar y del cuidarse.

Lo malo, en cambio, es lo que se asocia a lo que nos daña, a lo que nos destruye, o a lo que no va en bien de las comunidades que habitan la aldea. Una posición apocalíptica - ¿o apocalipticalizante? - frente al planeta, es un modo de estar en el mundo moralmente inadecuada. Pero no solo aquello que podría acabarlo materialmente, como una explosión nuclear o una crisis financiera ocasionada por terroristas que estrellan aviones contra importantes y emblemáticos edificios (tema de la citada obra de Morin y Baudrillard). Arrojar basura al suelo, no ceder la silla azul a las ancianas en el transporte público o adelantarse en la fila del banco son acciones inmorales, actitudes catalogadas como malas -aunque sea más reconocido como más malo el terrorista que hace estallar un carro-bomba en el corazón de una ciudad, que el joven-infante que asesina tras robar un teléfono celular en la misma ciudad; el primero recibe la condición de terrorista, mientras el segundo es un homicida culposo-. El mal allí, es un asunto de disertación judicial y es, por tanto, del orden del lenguaje. Unos y otros casos demuestran modos incorrectos de ser ciudadano.
Son ideas contrarias a la ciudadanía a la habitación de la aldea global, en el sentido de McLuhan (1994).

Sin embargo, la constitución del Estado moderno, analizada en no pocos trabajos de Michel Foucault, como es el caso de la genealogía del racismo (Dussel, 2003), se fundamenta en el despliegue de unas técnicas para el gobierno de la población, en cuyo centro está un modo del poder que no deviene de los usos habituales que hace la historia tradicional, en tanto la toma de decisiones desde arriba, sino más bien en una realización que está dada "en el nivel de los cuerpos, en el de las conductas y actuaciones materiales" (p. 18), es decir, desde abajo (Foucault, 2006; 2007). En otras palabras, una tesis que se fundamenta en la constitución del estado moderno, como un conjunto de prácticas que se articulan desde una perspectiva de gobierno liberal, que es en consecuencia, económico. Podemos afirmar, pues, que en una genealogía del presente, sería natural considerar una redistribución de las prácticas sociales, que se sitúa en una variación constante del poder, lo cual implica una perspectiva moral en transformación permanente. Esto si, de acuerdo con Castro (2012), el problema del poder en Foucault es, finalmente "un modo de afrontar el problema del sujeto" (p. 410), una ruta de entrada a la producción moderna de subjetividad.

Para principios del siglo XX, cierto sector de la moral se asoció con 
los usos y costumbres a propósito del cuerpo. Un cuerpo exhibido con más actitud explícita que erótica (o sea, menos en el sentido barthesiano del desocultamiento), se consideró como un comportamiento inmoral por parte del individuo responsable de tal acción. Algunas partes del cuerpo se constituyeron en prohibidas y su uso público, inmoral. Allí la moral es un asunto corpóreo, y se inscribe en un acervo opuesto diametralmente, por ejemplo al esbozado por Foucault, como el "nacimiento de una moralidad particular" (Dussel: 2003, 24), en tanto reflexión sobre la sexualidad, el deseo y el placer, en la línea de las claves para el autogobierno, rasgo fundamental en la pregunta por el poder. Esa moralidad particular puede asemejarse a la idea de la transvaloración en Nietzsche, que más adelante retomaremos.

Hablamos, pues, de al menos dos tipos de moral. Una que se vincula con la oposición moral/inmoral, bueno/malvado, y otra que se sitúa en una producción de subjetividad en clave ecológica, del autocuidado y cuidado del planeta, de la aldea. Esta última idea, enrostrada de manera recurrente en los discursos, a propósito de los derechos (de los hombres, de las mujeres, de los grupos, de los niños y hasta del medio ambiente), se encuadra en un tipo de subjetividad que es propia del Estado moderno. Las prácticas liberales, o la gubernamentalidad liberal (Marín y Noguera, 2011), nos proponen una construcción de la moralidad que se vincula con las condiciones que tienen los individuos para cuidar de sí, de los otros y del planeta. Se trata de la noción de comunidad, ampliamente promulgada en los discursos educativos contemporáneos, y de la noción de la competencia para habitar en un mundo que funciona cada vez más en tanto unidad, en tanto globalidad.

La escuela es, entre tanto, el lugar más indicado para ayudar a los sujetos a instalarse en los caminos de aquello que es bueno. Al menos esa es la idea más reciente que reposa sobre ella. Cuando la moral fue un asunto restringido al cuerpo, por ejemplo, entonces la escuela se configuró como el espacio llamado a dar preceptos a los infantes sobre el uso social de sus cuerpos. La idea de la virtud de mediados del siglo XX, estaba más relacionada con ese deseable uso del cuerpo, que con las ideas referidas a la instrucción, al trabajo y a la formación del carácter pestalozzianos de principios del siglo XIX.

En la segunda moral, aquella del discurso del cuidado, la escuela tiene hoy un perfil demarcado: se proponen proyectos pe- 
dagógicos (obligatorios, en el caso colombiano) con objetivos ambientalistas, con el propósito de generar conciencia, a los estudiantes en el cuidado del entorno -ien nombre del planeta!-, de sí mismos y de los otros. En otros casos, se formulan iniciativas de carácter pedagógico, en procura de desarrollar competencias ciudadanas, esto es, modos de saber hacer cosas para habitar bien el mundo. Las evaluaciones externas, aquellas que se practican como rasgo fundamental de calidad -otro síntoma de la planetizaciónde los llamados sistemas educativos, se erigen como simulacros que dan cuenta de un cierto deber ser de esesaber-hacer para estar en el planeta. Tal vez uno de los mejores lugares para continuar el proyecto nietzscheano de levantar una genealogía de la moral es justamente la escuela, o más ampliamente diríamos en la era contemporánea, en las sociedades educadoras, concepto que ha venido circulando con fuerza en los últimos tiempos.

\section{Sociedad educadora}

La delimitación de la pedagogía como campo de saber, ha sido discutida en las últimas décadas por grupos e investigadores, como es el caso del Grupo de Historia de la Práctica Pedagógica en Colombia. Parte de sus trabajos, especialmente aquellos realizados por Olga Lucía Zuluaga (2007), han asumido "el período comprendido entre Come- nio y Herbart como el lapso de conformación del horizonte conceptual de la pedagogía" (p. 99), y el auge del campo, especialmente a partir de la segunda mitad del siglo XX, como consecuencia de "las necesidades de formación de maestros que los nuevos aparatos de instrucción pública requirieron como parte de la expansión y consolidación de la escuela pública de masas" (Noguera, 2012, p. 19).

Para las últimas décadas del siglo $X X$, una vez Occidente comprendiera que la educación era un espléndido escenario para el gobierno de la población -aunque estas prácticas de hecho aparecieran, como nos ha propuesto Noguera (2012), desde los albores de la modernidad-, el mundo planetizado sufriría una suerte de pedagogización y entonces el acto educativo no se restringiría solamente a la escuela. Así, en el informe de Unesco (Faure et al., 1972) sobre los fines de la educación para la última parte del siglo $\mathrm{XX}$, presentado en 1972, se insistiría en la posibilidad de deslindar la educación de la escuela, acuñando por primera vez el concepto de ciudad educadora. Se dice en el informe: Henos aquí llevados más
allá de un simple cambio
de sistema, por radical
que sea este. Los que
cambian de naturaleza
son los términos mismos
de la relación entre so-
ciedad y educación. Una 
configuración social que situase a la educación en este lugar, que la otorgase este rango merecería un nombre propio: el de "Ciudad educativa". Su advenimiento solo sería concebible al término de un proceso de compenetración íntima de la educación y del tejido social, político y económico, en las células familiares, en la vida cívica. Implica que pueden ser puestas en todas las circunstancias a la libre disposición de cada ciudadano los medios de instruirse, de formarse, de cultivarse a su propia conveniencia, de tal suerte que el sujeto se encuentre respecto a su propia educación en una posición fundamentalmente diferente: la responsabilidad sustituyendo a la obligación. La educación no podrá, en esta perspectiva, constituir mañana sino un conjunto coordinado, en el que todos los sectores estarán estructuralmente integrados; será universalizada y continua; será desde el punto de vista de las personas, total y creadora: en consecuencia, individualizada y autodirigida. Será el soporte y el animador de la cultura, así como el motor de la promoción profesional. Este movimiento es irresistible e irreversible. Esta es la revolución cultural de nuestro tiempo. (Fauré et al., 1972, pp. 243-244).

Con este horizonte, el mundo contemporáneo de modo acelerado comenzaría con intentos múltiples de escenificar la educación en variados espacios, en los que la escuela se constituiría en un modo de núcleo, pero que en fin, se articularía con otros lugares de la ciudad, para dejarnos una vez más la sensación vigente de unidad que ha demandado la planetización. La educación sería un asunto de todos, para posicionarse incluso como un derecho de la humanidad, equiparable con el de vivir.

Entre el final del siglo XX y el comienzo del XXI, aparecerían conceptos y prácticas como ciudad educadora, sociedad educadora, sociedad de la información, e incluso, sociedad del aprendizaje", todos ellos como síntomas de la proliferación pedagógica, en una especie de actualización del ideal pansófico que trazará Comenio en su didáctica. La expresión artística no sería ajena a estos afanes, y es precisamente en este estado de cosas que se hizo posible la aparición de relatos que en mucho son reflejo de cuanto acontece en el mundo político, de la ideología, de la cultura y, por supuesto, de la educación. Este es el caso de Dogville, producto artístico del siglo XXI, del que nos ocuparemos en lo que sigue. 


\section{Dogville como totalidad:}

\section{una sociedad educadora}

Hemos esbozado dos ideas iniciales sobre una cierta actualidad de la moral y su posible contexto en los que identificamos como la sociedad educadora, en un ámbito de planetización. Quizá se aspire con estas ideas a apuntarle a las prácticas contemporáneas que dan cuenta de la moral en tanto su uso y, por tanto, articulada a los modos en que mutan los mecanismos del poder, en perspectiva foucaultiana. Para el efecto, avanzaremos en algunas reflexiones situadas en la película Dogville, una obra surgida en pleno siglo XXI, que ha sido posible en este momento de la historia, porque es precisamente en el presente que se han dado las condiciones para la producción de relatos enmarcados en estas ideas acerca de la moral con marco de referencia en el mundo como unidad. No interesa la noción de autor u obra, sino precisamente aquello que se dice (y lo que no se dice) en los discursos y las prácticas que se encarnan en ella.

Dogville es la primera película de una trilogía que el controvertido director danés Lars von Trier, precursor del movimiento Dogma 95, estrenó en 2003. La trilogía, titulada Estados Unidos: tierra de oportunidades, gira alrededor de una propuesta narrativa que ofrece la radiografía de sociedades de principios del siglo $X X$, que regulan la vida comunitaria desde la noción del trabajo. Se trata de personajes sin mayores matices, que componen familias que forman parte de poblaciones pequeñas; para el caso de la primera entrega, el pueblo de Dogville, y para el caso de la segunda, la población de Manderlay. La tercera parte de la trilogía, bautizada Washington, aún no ha sido estrenada.

Dogville se constituye en una metáfora que ridiculiza espléndidamente la idea de la comunidad, en este caso no del planeta, sino de una región que bien puede ser el mundo globalizado. No es gratuito que el director danés haya escogido a Estados Unidos como el escenario de su narración, al constituirse esta en la nación emblemática de los valores de la planetización, que hemos considerado a lo largo de esta exposición.

Grace, interpretada por la también emblemática actriz de Hollywood, Nicole Kidman, llega a Dogville huyendo del inmoral comportamiento de su padre, quien es un gánster arrogante, como recurrentemente señala la voz en off que acompaña la construcción del relato. Tom, por su parte, es un joven filósofo y escritor, sin mayor instrucción, pero con suficiente soberbia como para pretender ser un observador acertado y muy crítico de su comunidad. Los demás habitantes de Dogville son seres despreciables por su carácter egoísta y por llevar adelante una vida mediocre que no les permite ver más allá de los límites de su propio pueblo. Sin embar- 
go, son seres aceptablemente morales, en tanto son capaces de la vida comunitaria, al menos en la acepción de la moral que se esbozó en apartados anteriores. Son individuos con capacidad -competencia- para ser vecinos sin asesinarse unos a otros, incluso sin robarse entre sí: "en la historia de Dogville jamás hubo un robo", nos dice la voz en off, pues fundamentalmente se trataba de gente buena, con algunas faltas -io pecados?-, que es propio de cualquier ser humano, como el mismo Tom reconoció el día que Grace apareció en el pueblo, en plena huida de los gánsteres.

Grace contará con nueve capítulos -así se distribuye la narración en la película-, para convencer a los habitantes de Dogville de su capacidad para insertarse en la vida de la comunidad, para lo cual realiza una serie de trabajos cotidianos que la muestran como competente para ser miembro reconocido del pueblo, sin ser expulsada. Tom se erige como el inspector permanente de los progresos de Grace en su relación con los demás habitantes, y se autoproclama como el líder que es capaz de confrontar a la comunidad, siempre desde reflexiones venidas desde su convicción moral que, en apariencia, es compartida por los pobladores de Dogville. El pueblo se sostiene desde una serie de preceptos morales que regulan la vida en comunidad y que ofrecen límites a los rasgos de personalidad de cada habitante por separado, y de cada familia en conjunto.

Un primer giro en la narración se da en el momento en que Grace, tras trabajar arduamente por el bienestar de las familias (apoya labores de aseo en las casas, de siembra y cosecha de alimentos, funge como acompañante en las tardes vacías del vecino que es ciego, cuida los niños maleducados de una familia numerosa), es violentada de maneras diversas. Es objeto de humillación y de burla por parte las mujeres, y violada por los hombres; primero por Chuck, y luego de manera sistemática por los demás. Es chantajeada por los habitantes de Dogville, pues finalmente se trata de una fugitiva de los gánsteres, razón por la que incluso es tratada como prisionera, a la que le hacen juicios colectivos que terminan con la singular sentencia de encadenarla en las noches, y portar un grillete en el cuello durante el día.

En el último capítulo, con el giro final, Grace es encontrada por su padre, el gánster. De manera sorpresiva, Grace le solicita que asesine a hombres, mujeres y niños y que queme los restos 
del miserable pueblo, pues sin esa población, "el mundo sería un lugar mejor".

Dogville se constituye en lo que podríamos llamar en la era contemporánea, una sociedad educadora, en tanto sus miembros se muestran en aparente capacidad para la convivencia armónica en un mundo que exige equilibrio por parte de los sujetos que viven en comunidad, además de una noción construida a propósito del desarrollo. Este último, está asociada, en Dogville, a la satisfacción de las necesidades básicas de los sujetos, tal como podemos considerar el primer renglón de una sociedad contemporánea, inscrita en los pilares de la planetización.

Dogville, como sociedad educadora, cuenta además con la posibilidad del aprendizaje, idea restringida a la acción moral, a partir de los acuerdos construidos comunitariamente. Gravita allí una noción del aprendizaje emparentada con el concepto experiencia, que fuera muy útil en las reflexiones de la psicología al servicio de las ciencias de la educación, de principios del siglo XX (Hilgard y Bower, 2000; Dewey, 1967). Se trata de una concepción de la experiencia, en tanto posibilidad para acceder al mundo desde los actos cotidianos que invocan nociones como el crecimiento; al decir de John Dewey, en varios de sus trabajos, la experiencia se constituye en pilar de la acción educativa, pues a través de ella los sujetos construyen relaciones con el mundo y la realidad, cada vez de manera más adecuada, a las exigencias de los contextos y de los otros sujetos. Las sanciones impuestas de modo creciente a Grace, así como la idea del castigo, emergen con naturalidad en las rutinas de los pobladores, y esto aparece como un efecto de los acuerdos que en comunidad se van construyendo a lo largo del relato.

\section{Grace: ¿más o menos Nietzsche?}

La idea de la transvaloración, definida por Nietzsche (2009) como la inversión "de la identificación aristocrática de los valores (bueno $=$ noble $=$ poderoso $=$ bello $=$ feliz = amado de Dios)" para más bien otorgar estatus de bueno a "los miserables, los pobres, los impotentes, los que sufren, los indigentes, los enfermos" (p. 46), es una idea que quiso explorar ampliamente Lars von Trier con Dogville: Dogville es "Villa-perro", quizás porque al final solo el perro Moisés -única mascota en el pueblo- es el sobreviviente accidental, pero también elegido; quizás porque igual que el Moisés bíblico, es el digno de ser rescatado de esas aguas nocivas de la comunidad.

La transvaloración en Dogville, sin embargo, no es un asunto únicamente de los habitantes del pueblo. Es una experiencia quizás aún más visible en Grace. 
Inicialmente, la capacidad para el perdón y la condescendencia, es el atributo moral más visible en la nueva habitante de Dogville. Sin embargo, esa capacidad moralmente adecuada es sancionada por su propio padre al final de la historia, como una actitud arrogante. Lo arrogante es, pues, una actitud moralmente inadecuada. Tal vez por esto, hoy no podemos hablar de inmoralidad o de antivalores, como es muy frecuente en espacios como los escolarizados. Hoy hablamos de valores que se transforman, de valores que son distintos a los otros, en especial de aquellos que no son recientes, aquellos practicados por las otras generaciones y que hoy poco tiene que ver con la escuela.

Los habitantes del pueblo son gente buena, que reafirma su bondad a través de la historia: han acogido a una fugitiva, y mediante un acto de fe la cobijan en sus predios. Por supuesto, parece ser que una acción generosa, aunque signada por principios reconocibles como buenos (el hecho de acoger a una fugitiva partiendo de la presunción de inocencia hasta que no sea demostrado lo contrario), merece ser retribuida por otra $u$ otras acciones igualmente buenas.

Sin embargo, en un sentido griego, como diría Nietzsche (2009), Dogville es un pueblo de infelices y, por tanto, de sujetos malos. Infelicidad y maldad son dos ideas que suenan "al oído griego con un tono único" (p. 54) y en ese tono es preponderante lo infeliz. En el capítulo primero de la película, cuando Tom presenta a Grace el pueblo y observa la decadencia y la infelicidad de cada uno de sus habitantes, o cuando Chuck, en medio de su resistencia a la presencia de Grace, le pregunta si ya ha notado la farsa, no la suya, sino aquella de las vidas de los vecinos de Dogville.

Grace construye su acervo moral como la hacían los nobles que en su genealogía cita permanentemente Nietzsche (2009), mientras que los demás habitantes del pueblo lo hacen con una cierta estética de los esclavos. "Mientras que toda moral noble nace de un triunfante sí dicho a sí mismo, la moral de los esclavos dice no, ya de antemano, a un 'fuera', a un 'otro', a un 'no-yo'; y ese no es lo que constituye su acción creadora" (p. 50). Por definición, dice Nietzsche, los nobles hacían bueno, pues esa es justamente la calidad de su condición. La acción de los esclavos, en cambio, se gesta desde el resentimiento, desde una suerte de deuda sistemática que les tiene reservada el mundo y desde una noción de lo malvado que no es solamente distinto a lo bueno, sino que es trazo inequívoco del fin, de cualquier modo de final y de finales del mundo. 
Grace acepta las condiciones de pago por ser recibida en Dogville. Pasa de no conocer las actividades propias del trabajo, a ser explotada de todos los modos posibles. Se hace esclava en las acciones, aunque es de nobles su perspectiva moral. $Y$ allí se inscribe precisamente la transvaloración nietzscheana: no se trata de la actitud final de Grace al querer exterminar incluso con saña- a los habitantes de Dogville para "hacer del mundo un lugar mejor", sino desde la misma acción de la comunidad.

La transvaloración allí está en que lo bueno no es necesariamente lo bello, sino que más bien lo bueno se vincula con lo que es justo. La justicia de la comunidad reside en el hecho de aceptar a Grace, y en el curso de esa aceptación es bello y justo, por demás, que Grace pague por ella al precio de su propia degradación. "Lo bueno allí" es justamente lo que es malo en la lógica de las otras morales, en las que desde ninguna perspectiva sería aceptable la explotación, la discriminación o la puesta en condición indigna de sujeto alguno, como puede leerse en diversas declaraciones de los derechos, o como puede leerse en los manuales de convivencia escolares. Las declaraciones acerca de valores tales como la equidad, la justicia o la igualdad, forman parte de los discursos totalizadores que aspiran al mundo como unidad, aun cuando la perspectiva moral, para la que incluso se educa, proceda del reordenamiento discursivo propio de la globalización económica.

\section{Referencias}

Castro, E. (2012). El vocabulario de Michel Foucault. Un recorrido alfabético por sus temas, conceptos y autores. Recuperado el 30 de junio de 2012 de: http:// es.scribd.com/doc/11414155/ Castro-Edgardo-El-Vocabulariode-Mfoucault

Dewey, J. (1967). Experiencia y educación. Buenos Aires: Losada.

Dussel, I. (2003). "Foucault y la escritura de la historia: reflexiones sobre los usos de la genealogía". En: Revista Educación y Pedagogía. Medellín: Universidad de Antioquia. Vol. XV, No. 37 (septiembre - diciembre). pp $13-31$.

Faure, E. et al. (1972). Aprender a ser. La educación del futuro. Informe para Unesco. Madrid: Alianza.

Foucault, M. (1979). Nietzsche, la genealogía, la historia. En: $M$. Foucault. Microfísica del poder (pp. 7-29). Madrid: Ediciones La Piqueta.

Foucault, M. (1996). La verdad y las formas jurídicas. Barcelona: Gedisa.

Foucault, M. (2004). La arqueología del saber. Buenos Aires. Siglo XXI Editores.

Foucault, M. (2006). Seguridad, territorio y población. Buenos Aires: Fondo de Cultura Económica.

Foucault, M. (2007). Nacimiento de la biopolítica. Buenos Aires: Fondo de Cultura Económica. 
Hilgard, E. y Bower, G. (2000). Teorías del aprendizaje. México: Trillas.

Mcluhan, M. (1994). La aldea global: transformaciones en la vida y los medios de comunicación mundiales en el siglo XXI. Barcelona: Agostini.

Morin, E. y Baudrillard, J. (2004). La violencia del mundo. Buenos Aires: Paidós.

Marín, D. y Noguera, C. (2011). "Educar es gobernar". En: R. Cortés (coord.). Gubernamentalidad y educación. Discusiones contemporáneas (pp. 127 - 151). Bogotá: IDEP.

Nietzsche, F. (2009). La genealogía de la moral. Madrid: Alianza Editorial.

Noguera, C. (2012). El gobierno pedagógico. Del arte de educar a las tradiciones pedagógicas. Bogotá: Siglo del Hombre Editores.

Von Trier, L. (2003). Dogville. Película.

Zuluaga, O. (2007). "Otra vez Comenio". En: Revista Educación y Pedagogía. Medellín: Universidad de Antioquia. Vol. XIX, No. 47 (enero - abril), pp 99 - 118. 